\title{
Sex Differences in Associations Between CYP2D6 Phenotypes and Response to Opioid Analgesics
}

This article was published in the following Dove Press journal:

Pharmacogenomics and Personalized Medicine

\author{
Guilherme S Lopes (iD) ${ }^{1,2}$ \\ Suzette J Bielinski \\ Ann M Moyer (D) ${ }^{3}$ \\ John Logan Black III \\ Debra J Jacobson' \\ Ruoxiang Jiang' \\ Nicholas B Larson (DD \\ Jennifer L St Sauver ${ }^{2}$ \\ 'Division of Biomedical Statistics and \\ Informatics, Department of Health \\ Sciences Research, Mayo Clinic, \\ Rochester, MN, USA; ${ }^{2}$ Division of \\ Epidemiology, Department of Health \\ Sciences Research, Mayo Clinic, Rochester, \\ MN, USA; ${ }^{3}$ Division of Laboratory Genetics \\ and Genomics, Department of Laboratory \\ Medicine and Pathology, Mayo Clinic, \\ Rochester, MN, USA
}

Background: Several small studies have previously investigated associations between the cytochrome P450 2D6 (CYP2D6) metabolism and response to opioids. We used a large sample of patients to study associations between CYP2D6 phenotypes and estimated CYP2D6 enzymatic activity scores with pain control and adverse reactions related to codeine and tramadol use. We conducted additional analyses to determine whether our results were consistent among men and women.

Methods: We used data from 2,877 participants in the RIGHT Protocol who were prescribed codeine and/or tramadol between 01/01/2005 and 12/31/2017 and who were not prescribed CYP2D6 inhibitors within 1 year prior to the opioid prescription. CYP2D6 phenotype categories were condensed into four groups: (1) Ultra-rapid and Rapid ( $\mathrm{n}=61)$, (2) Normal and Intermediate to Normal $(n=1,448)$, (3) Intermediate and Intermediate to Poor $(n=1,175)$, and (4) Poor metabolizer status $(n=193)$. Opioid-related outcomes included indications of poor pain control or adverse reactions related to medication use. We modeled the risk of each outcome using logistic regression, adjusting for age, sex, race, and ethnicity.

Results: The results revealed a trend from poor to ultra-rapid and rapid CYP2D6 phenotypes in which the risk of adverse reactions incrementally increased and the risk of poor pain control incrementally decreased. This trend reached statistical significance among female (but not male) participants. Among normal and intermediate to normal metabolizers, a larger proportion of women experienced adverse reactions relative to men.

Discussion: We replicated and extended the findings of previous research indicating associations between CYP2D6 phenotypes and response to opioids. In addition, the observed associations were stronger in women than in men. We recommend sex differences to be factored in future research investigating associations between pharmacogenomics and response to medications.

Keywords: opioids, codeine, tramadol, pharmacogenomics, sex differences, CYP2D6

\section{Introduction}

Opioids are among the most commonly prescribed medications in the United States, with $17 \%$ of the population filling at least one prescription in $2017 .{ }^{1}$ Despite this widespread use of opioids, previous research has documented important individual differences in effectiveness in relieving pain as well as adverse reactions and addiction. $^{2-4}$ Understanding the individual characteristics affecting response to opioids may allow clinicians to individualize treatments, thereby optimizing effectiveness and reducing adverse reactions related to opioid use.

Variation in genes encoding enzymes important in the metabolism of opioids leads to different individual responses to these medications. For codeine and
Correspondence: Guilherme S Lopes Informatics, Department of Health Sciences Research, Mayo Clinic, 200 First Street SW, Rochester, MN 55905, USA

Tel + I 5074226094

Email lopes.guilherme@mayo.edu 
tramadol, the cytochrome P450 2D6 (CYP2D6) enzyme is involved in prodrug conversion of these opioids to their active metabolites. Genetic variation in CYP2D6 directly affects CYP2D6 enzyme activity. ${ }^{5}$ An individual's CYP2D6 activity can be predicted based on his or her CYP2D6 genotype (eg, poor metabolizer). Individuals with a CYP2D6 ultra-rapid metabolizer phenotype may be more likely to experience adverse reactions with some opioids as they would convert prodrugs - such as tramadol and codeine-more efficiently into morphine. ${ }^{6}$ By contrast, individuals with a CYP2D6 poor metabolizer phenotype would have a more limited conversion of these prodrugs to their active metabolites and may experience less pain relief relative to individuals with a CYP2D6 normal metabolizer phenotype. ${ }^{7}$

Several small studies have previously investigated associations between CYP2D6 phenotypes and response to opioids, although findings have been limited. For example, previous research investigating the response to codeine use among 45 women reported that $100 \%$ of poor metabolizers experienced no analgesia, whereas $67 \%$ of ultra-rapid metabolizers reported immediate pain relief, but also reported adverse reactions. ${ }^{8}$ A clinical trial investigating the effects of CYP2D6-guided opioid prescribing on pain control found that intermediate and poor metabolizers prescribed genotype-appropriate doses of tramadol and/or codeine $(n=45)$ had greater improvement of pain intensity in the CYP2D6-guided versus usual care arm. ${ }^{9}$ Our own investigations of patient response related to codeine, tramadol, hydrocodone, and oxycodone in a cohort of 257 patients found that more than $30 \%$ of those with a CYP2D6 poor or ultra-rapid phenotype experienced either adverse reactions or lack of pain relief related to opioid use. ${ }^{10}$ In short, these results suggest poor pain control in individuals with CYP2D6 poor metabolizer status and more adverse reactions in individuals with CYP2D6 ultra-rapid metabolizer status.

Previous studies investigating associations between CYP2D6 phenotypes and response to opioids have contributed to advancing pharmacogenomics-guided prescribing guidelines. ${ }^{11}$ However, these previous studies have important limitations. First, low event rates and small sample sizes result in a large degree of effect estimate uncertainty, making the findings difficult to generalize to the general population. ${ }^{8-10}$ In addition, our previous research collapsed ultra-rapid metabolizers and poor metabolizers when comparing to normal metabolizers due to phenotype rarity, limiting the interpretation of results. ${ }^{10}$ Third, previous research has used different classifications of CYP2D6 phenotypes, such that results may not be comparable across studies. ${ }^{11-13}$ Fourth, pain relief and adverse reactions related to opioid medications occur differently in men and women, but very few studies have investigated sex differences in response to opioids. ${ }^{14,15}$ In particular, previous research has reported sex differences in the formation of active opioid metabolites and in clinical responses to codeine and tramadol, ${ }^{16,17}$ but it is currently unknown whether there is an interaction between sex and CYP2D6 phenotypes affecting response to opioids. To overcome previous limitations, we studied associations between both CYP2D6 phenotypes and estimated CYP2D6 enzymatic activity scores with pain relief and adverse reactions related to codeine and tramadol use in a large sample of patients. In addition, we conducted a secondary analysis to determine if our results were consistent among men and women.

\section{Materials and Methods}

\section{Study Participants}

Details of the RIGHT Protocol study have been previously reported. ${ }^{13,18,19}$ Briefly, 10,074 participants in the Mayo Clinic Biobank were invited and agreed to participate in a study of pre-emptive genotyping. ${ }^{20}$ Participants completed a questionnaire that included demographic information and contributed a blood sample that was used to sequence 70 pharmacogenomic (PGx) genes, including CYP2D6. At the time of enrollment, participants in the RIGHT study provided written informed consent to allow their PGx genetic data and electronic health record data to be used for research in future studies. This study was approved by the Mayo Clinic and Olmsted Medical Center Institutional Review Boards and was conducted in accordance with the Declaration of Helsinki.

We used the Rochester Epidemiology Project ${ }^{21}$ research infrastructure to identify all participants in the RIGHT Protocol who were prescribed codeine or tramadol between 1/1/2005 and 12/31/2017 from a local health-care provider. Specifically, we used RxNorm - a national database of normalized names for clinical drugs - to identify all codes for prescriptions that included the ingredients codeine or tramadol. We excluded formulations intended primarily to treat cough (eg, guaifenesin/codeine). Participants who had a prescription for codeine or tramadol between 1/1/2004 and 12/31/2004 were excluded to focus on initiators of codeine/tramadol use. A complete list of medications considered is shown in Table S1. 


\section{CYP2D6 Sequencing}

For non-pilot RIGHT participants, the Clinical Laboratory Improvement Amendments (CLIA)-certified and College of American Pathologists (CAP)-accredited Baylor College of Medicine's Human Genome Sequencing Center Clinical Laboratory sequenced 70 PGx genes using the PGRNseq version 3 targeted capture sequencing panel, including the entire $\sim 31 \mathrm{~kb}$ region for CYP2D6 and both of the nearby pseudogenes. Data were then transferred to the Mayo Clinic Personalized Genomics Laboratory for interpretation. Genotypes were determined using software developed and clinically validated at Mayo Clinic. This software circumvents some of the challenges of short-read NGS technology and produces allele calls based on the definitions publically available in PharmVar (www.pharmvar.org). The software looks at copy number variation by analyzing read depth of the promoters and exons in CYP2D7 and CYP2D7 and compares the output to known $C Y P 2 D$ loci arrangements. CYP2D6 genotypes were also determined based on variants detected in the coding regions, promoter, and splice junctions. Novel variants were evaluated for their potential impact on CYP2D6 activity and those that were classified as variants of uncertain significance or likely to impact activity were reported. Samples were reflexed through a cascade similar to that used for the pilot participants as needed to deconvolute difficult genotypes that could not be determined by the software. $^{22,23}$

\section{CYP2D6 Phenotypes}

CYP2D6 phenotypes were classified based on estimated activity scores of each CYP2D6 allele. We estimated activity scores based upon a review of the extensive literature on CYP2D6 and the PharmVar.org website. Each allele has an activity score that varied from $0\left(\mathrm{eg},{ }^{*} 6,{ }^{*} 4\right)$ to $3\left(\mathrm{eg},{ }^{*} 1 \times 3\right)$. The activity score of a CYP2D6 genotype is the sum of activity scores of the two homologous alleles. For example, if an individual carries one copy of allele * 1 with an activity score of 1 , and additionally carries one copy of allele $* 9$ with activity score of 0.5 , the resulting genotype $* 1 / * 9$ has an activity score of 1.5 . For this study, we used the CYP2D6 phenotype classification that is in use clinically at Mayo Clinic (CYP2D6 genotype activity scores denoted as " $\mathrm{x}$ "): ultra-rapid ( $x \geq 3$ ), rapid (formerly known as extensive to ultra-rapid) $(2.25<x<3)$, normal $(1.75 \leq x \leq 2.25)$, intermediate to ultra-rapid $(0.75<\mathrm{x}<3)$, intermediate to normal $(1.25<\mathrm{x}<1.75)$, intermediate $(0.75 \leq \mathrm{x} \leq 1.25)$, poor to intermediate $(0.25 \leq \mathrm{x}<0.75)$, and poor CYP2D6 metabolizer status $(\mathrm{x}<0.25)$.

\section{Outcomes}

To identify potential opioid-related outcomes, electronic health records were initially screened by natural language processing techniques. Specifically, sentences including the opioid name (codeine, tramadol, and trade names for medications including these ingredients) and containing keywords related to opioid effectiveness or presence of adverse reactions due to opioid use were extracted from the clinical notes within 6 weeks of the initial prescription date. All sentences were then reviewed by the study personnel (JLS and a trained nurse abstractor), and classified as adverse effect (yes/no), poor pain control (yes/no), or other. For example, sentences such as "Patient has been taking Ultram but continues to complain of severe neck pain" were classified as "poor pain control". Medical notes that indicated an adverse reaction related to the medication use (eg, vomiting, nausea, rash, itching, throat swelling, constipation) were classified as "adverse reaction". This process is summarized in Figure S1. All records were reviewed without knowledge of the patient CYP2D6 phenotype. Patients were classified as having experienced an adverse reaction or poor pain control if specific medical record text related the codeine or tramadol prescription to these outcomes.

\section{CYP2D6 Inhibitors}

Concurrent use of CYP2D6 inhibitor medications may decrease or prevent the conversion of opioid medications to morphine. Therefore, we excluded participants who were prescribed a medication that was a strong or moderately strong inhibitor of CYP2D6. ${ }^{24}$ The list of inhibitors considered for this study is shown in Table S2. Because most of these prescriptions are intended to be taken long term, we considered individuals exposed to a CYP2D6 inhibitor if the prescription occurred within 1 year prior to the opioid prescription.

\section{Statistical Analyses}

For analysis, the CYP2D6 phenotype categories were condensed into four groups to mirror the phenotype prediction system recommended by the Clinical Pharmacogenetics Implementation Consortium (CPIC): (1) Ultra-rapid and Rapid ( $\mathrm{n}=61)$, (2) Normal and Intermediate to Normal $(\mathrm{n}=1,448)$, (3) Intermediate and Intermediate to Poor $(\mathrm{n}=1,175)$, and (4) Poor metabolizer status $(\mathrm{n}=193)$. To 
facilitate comparison of results across studies, we also investigated the associations between CYP2D6 activity scores and response related to opioid use. We collapsed participants prescribed codeine and/or tramadol to increase stability of estimates. However, because the pharmacodynamic profiles of codeine and tramadol are different, ${ }^{25}$ we also conducted sensitivity analysis including participants who were prescribed tramadol only $(n=2,085)$. Limited sample sizes precluded us from investigating participants prescribed codeine only $(\mathrm{n}=$ 481). Baseline patient characteristics were summarized using counts and percentages for nominal variables, and mean, standard deviation, median, and quartiles for real-valued variables. We calculated differences between CYP2D6 phenotypes in age and CYP2D6 activity scores using Kruskal-Wallis tests, and in the proportions of sex, race, ethnicity, and prescriptions using $\chi^{2}$ tests.

First, we conducted univariate analyses to investigate whether sex, age, race, and ethnicity are associated with the risk of poor pain control and adverse reactions. We adjusted effect estimates and $p$-values for these covariates in subsequent analyses. Next, we modeled the risk of each outcome using Firth's logistic regression, treating CYP2D6 phenotypes as categorical variables and the normal and intermediate to normal metabolizer phenotype as the reference group. We used Firth's logistic regression to account for separation issues caused by the inclusion of covariates. We investigated trends of risk of poor pain control and adverse reactions across CYP2D6 phenotypes. We also included interaction terms between sex and CYP2D6 phenotypes. Finally, we conducted these analyses by sex separately, adjusting effect estimates and $p$-values for age, race, and ethnicity. As different methodologies for phenotype categorization have been proposed, ${ }^{11-13}$ we also modeled associations between CYP2D6 activity scores and each outcome using logistic regression after verifying that CYP2D6 activity scores and log-odds were linearly associated. A two-sided significance threshold of $p<0.05$ was adopted for all analyses.

\section{Results}

Among 10,074 RIGHT non-pilot participants, 3911 (39\%) participants received at least one prescription of codeine or tramadol between 1/1/2005 and 12/31/2017 and had not received at least one prescription in 2004 . We excluded 39 (1\%) participants because their CYP2D6 phenotype classification was not clear (eg, interpretation of activity score resulted in an intermediate to ultra-rapid phenotype due to presence of a rare genetic variant).
From the remaining 3,872 participants, we excluded 995 (26\%) because they were prescribed a CYP2D6 inhibitor within 1 year prior to the opioid prescription. The final sample included 2,877 participants. Participant characteristics are shown in Table 1 . Of these, $61(2.1 \%)$ were ultra-rapid or rapid metabolizers, 1,448 (50\%) were normal or intermediate to normal metabolizers, 1,175 (41\%) were intermediate or poor to intermediate, and $193(6.7 \%)$ were poor metabolizers. In addition, 481 (16.7\%) participants were prescribed codeine only, $2,085(72.5 \%)$ were prescribed tramadol only, and 311 (10.8\%) were prescribed both opioids. Among the 198 participants with adverse reactions, 61\% experienced gastrointestinal issues such as nausea, vomiting, and constipation. Itching and rash were present in $7.1 \%$ of the participants, and the remaining participants had other or unspecified reactions (eg, fatigue, dizziness, anxiety). The proportions of CYP2D6 phenotypes were similar for sex, ethnicity, and type of prescription (ie, codeine, tramadol, or both opioids). In addition, age did not significantly differ between CYP2D6 phenotypes (Table 1).

The results of univariate tests indicated that older persons $(p=0.025)$ and women (relative to men; $p=0.002$ ) were more likely to experience adverse reactions. In addition, Blacks (compared to Whites; OR = 5.1 [1.1-24.1], $p=0.038$ ) were more likely to experience poor pain control (Table S3). For this reason, we included age, sex, race, and ethnicity as covariates in the subsequent regression models.

Next, we examined associations between CYP2D6 phenotypes and adverse reactions and poor pain control, controlling for age, sex, race, and ethnicity. Participants with a CYP2D6 ultra-rapid or rapid phenotype were least likely to have poor pain control $(1.6 \%)$, and participants with a CYP2D6 poor phenotype were most likely to have a poor pain control recorded $(5.2 \% ; p=0.13$; Figure 1$)$. Conversely, participants with a CYP2D6 ultra-rapid or rapid metabolizer phenotype experienced the highest rates of adverse reaction $(8.2 \%)$, and participants with a CYP2D6 poor metabolizer phenotype were least likely to have an adverse reaction $(5.7 \% ; p=0.11$; see Figure 1$)$. However, we note that the tests for trend were not statistically significant. Among participants who were prescribed tramadol only $(\mathrm{n}=2,085)$, we found a significant trend for poor pain control $(p=0.039)$ but not for adverse reactions $(p=0.69)$.

Analyses of CYP2D6 activity scores additionally excluded 89 participants with ambiguous scores (eg, carriers 
Table I Baseline Characteristics of Participants by CYP2D6 Metabolizer Phenotypes $(n=2,877)$

\begin{tabular}{|c|c|c|c|c|c|c|}
\hline \multirow[t]{2}{*}{ Characteristic } & \multicolumn{4}{|c|}{ CYP2D6 Metabolizer Phenotypes } & \multirow[t]{2}{*}{ Total } & \multirow[t]{2}{*}{$\mathbf{p}$} \\
\hline & $\begin{array}{l}\text { Ultra-Rapid and } \\
\text { Rapid N = 6I }\end{array}$ & $\begin{array}{l}\text { Normal and } \\
\text { Intermediate to } \\
\text { Normal } N=1,448\end{array}$ & $\begin{array}{l}\text { Intermediate } \\
\text { and } \\
\text { Intermediate to } \\
\text { Poor } N=1,175\end{array}$ & $\begin{array}{l}\text { Poor } \\
N=193\end{array}$ & & \\
\hline Total & $61(2.1)$ & $\mathrm{I}, 448(50)$ & $\mathrm{I}, \mathrm{I} 75(4 \mathrm{I})$ & $193(6.7)$ & 2877 & \\
\hline $\begin{array}{l}\text { Sex, } \mathbf{n}(\%) \\
\quad \text { Male } \\
\text { Female }\end{array}$ & $\begin{array}{l}24(2.0) \\
37(2.2)\end{array}$ & $\begin{array}{l}583(49) \\
865(5 \mathrm{I})\end{array}$ & $\begin{array}{l}494(42) \\
681(40)\end{array}$ & $\begin{array}{l}85(7.2) \\
108(6.4)\end{array}$ & $\begin{array}{l}1,186 \\
1,691\end{array}$ & 0.66 \\
\hline $\begin{array}{l}\text { Age } \\
\text { Mean } \\
\text { SD }\end{array}$ & $\begin{array}{l}63 \\
12\end{array}$ & $\begin{array}{l}61 \\
14\end{array}$ & $\begin{array}{l}61 \\
13\end{array}$ & $\begin{array}{l}63 \\
14\end{array}$ & $\begin{array}{l}61 \\
14\end{array}$ & 0.09 \\
\hline $\begin{array}{l}\text { Race, n (\%) } \\
\text { White } \\
\text { Black } \\
\text { Asian/Pacific } \\
\text { Islander } \\
\text { Unknown }\end{array}$ & $\begin{array}{l}53(2.0) \\
0(0.0) \\
0(0.0) \\
8\end{array}$ & $\begin{array}{l}1,355(50) \\
6(55) \\
18(78) \\
69\end{array}$ & $\begin{array}{l}\text { I,I I (4I) } \\
5(46) \\
5(22) \\
46\end{array}$ & $\begin{array}{l}179(6.6) \\
0(0.0) \\
0(0.0) \\
14\end{array}$ & $\begin{array}{l}2,706 \\
11 \\
23 \\
137\end{array}$ & 0.02 \\
\hline $\begin{array}{l}\text { Ethnicity, n (\%) } \\
\text { Non-Hispanic } \\
\text { Hispanic } \\
\text { Unknown }\end{array}$ & $\begin{array}{l}59(2.1) \\
2(5.9) \\
0\end{array}$ & $\begin{array}{l}\mathrm{I}, 428(50) \\
\mathrm{I} 7(50) \\
3\end{array}$ & $\begin{array}{l}\mathrm{I}, \mathrm{I} 60(4 \mathrm{I}) \\
\mathrm{I} 4(4 \mathrm{I}) \\
\mathrm{I}\end{array}$ & $\begin{array}{l}192(6.8) \\
1(2.9) \\
0\end{array}$ & $\begin{array}{l}2,839 \\
34 \\
0\end{array}$ & 0.39 \\
\hline $\begin{array}{l}\text { Prescription, n (\%) } \\
\text { Codeine only } \\
\text { Tramadol only } \\
\text { Both opioids }\end{array}$ & $\begin{array}{l}8(1.7) \\
47(2.3) \\
6(1.9)\end{array}$ & $\begin{array}{l}243(5 \mathrm{I}) \\
\mathrm{I}, 054(5 \mathrm{I}) \\
\mathrm{I} 5 \mathrm{I}(49)\end{array}$ & $\begin{array}{l}191(40) \\
854(41) \\
130(42)\end{array}$ & $\begin{array}{l}39(8.1) \\
130(6.2) \\
24(7.7)\end{array}$ & $\begin{array}{l}481 \\
2,085 \\
311\end{array}$ & 0.71 \\
\hline $\begin{array}{l}\text { CYP2D6 activity } \\
\text { score } \\
\text { Total } \\
\text { Mean } \\
\text { SD }\end{array}$ & $\begin{array}{l}58 \\
2.9 \\
0.4\end{array}$ & $\begin{array}{l}\mathrm{I}, 394 \\
\mathrm{I} .8 \\
0.3\end{array}$ & $\begin{array}{l}1,144 \\
0.9 \\
0.2\end{array}$ & $\begin{array}{l}192 \\
0 \\
0\end{array}$ & $\begin{array}{l}2,788 \\
1.4 \\
0.7\end{array}$ & $<0.001$ \\
\hline
\end{tabular}

of variants of unknown significance). Among the remaining participants $(\mathrm{n}=2,788), 115(4.1 \%)$ experienced poor pain control and $192(6.9 \%)$ experienced adverse reactions. CYP2D6 activity scores varied from $0(\mathrm{eg}, * 4 / * 5)$ to $4(\mathrm{eg}$, $* 1 / * 2 \mathrm{Ax} 3)$, with a mean of $1.36(\mathrm{SD}=0.65)$. Figure $\mathrm{S} 2$ displays activity scores of participants stratified by eventstatus for adverse reactions or poor pain control related to opioid use. Mean CYP2D6 activity scores of participants who experienced and did not experience poor pain control were $1.30(\mathrm{SD}=0.64)$ and $1.36(\mathrm{SD}=0.65)$, respectively ( Figure $\underline{\mathrm{S} 2}$ ). In addition, mean CYP2D6 activity scores of participants who experienced and did not experience adverse reactions were, respectively, $1.43(\mathrm{SD}=0.65)$ and $1.35(\mathrm{SD}=0.65)$. Next, we conducted a Wilcoxon rank-sum test with continuity correction and found that the median and shape of ranked CYP2D6 activity scores did not differ between participants who experienced versus did not experience poor pain control $(p=0.44)$ or adverse reactions $(p=0.066)$. After controlling for age, sex, race, and ethnicity, an increase in CYP2D6 activity scores was not significantly associated with risk of poor pain control $(p=0.32)$ or adverse reactions $(p=0.10)$.

Finally, it is currently unknown whether there are sex differences in the associations between pain control and adverse reactions related to use of opioid medications and CYP2D6 phenotypes, despite National Institutes of Health (NIH) guidelines for clinical research recommending sex differences to be factored into research design and statistical analysis (NIH, 2020). We therefore compared pain 


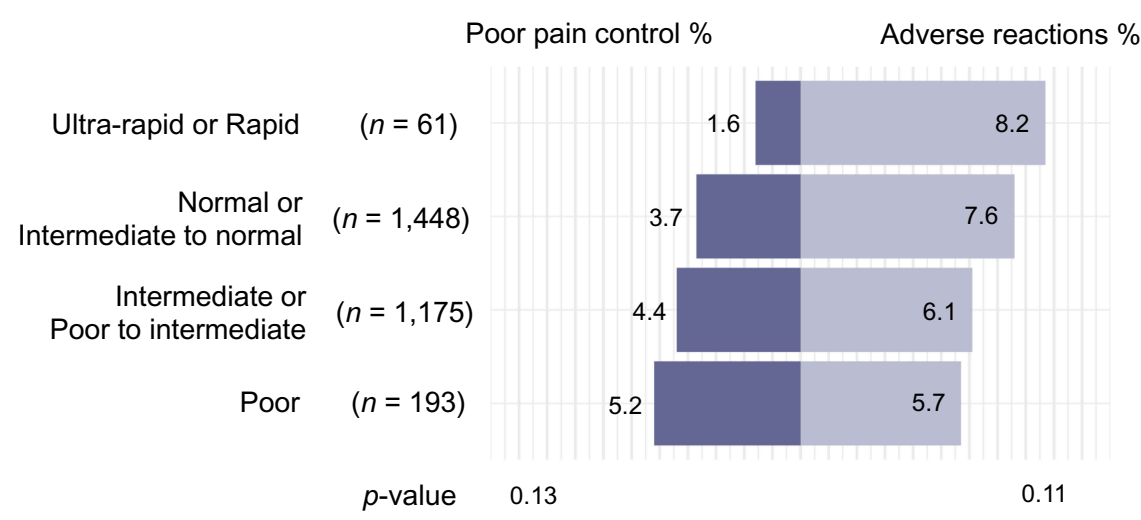

Figure I Adverse reactions and poor pain control following the prescription of codeine and/or tramadol among participants who were not prescribed CYP2D6 inhibitors $(n=2,877)$.

control and adverse reactions by CYP2D6 phenotypes in men and women separately. ${ }^{14,15}$ The results revealed a trend from poor to ultra-rapid and rapid CYP2D6 phenotypes in which the risk of adverse reactions significantly increased and the risk of poor pain control decreased for women ( $p=0.041$ and 0.040 , respectively), but not for men ( $p=0.95$ and 0.77 , respectively; see Figure 2 ). In addition, among normal and intermediate to normal metabolizers, approximately twice as many women (9.5\%) experienced adverse reactions compared to men (4.8\%), suggesting that sex modifies the association between CYP2D6 phenotypes and response to codeine and tramadol. The results including participants prescribed tramadol only $(n=2,085)$ revealed non-significant trends for men. For women, we found a trend of poor pain control ( $p=0.032$ ), while the trend of adverse reaction was non-significant $(p=0.15)$. However, we note that we did not observe a statistically significant interaction between sex and CYP2D6 phenotype. Figure S3 displays the associations between the CYP2D6 phenotypes among men and women separately prescribed codeine and/or tramadol or

\section{$A \operatorname{Men}(n=1,186)$}

Ultra-rapid or Rapid
$\begin{array}{r}\text { Normal or } \\ \text { Intermediate to normal }\end{array}$
$\begin{array}{r}\text { Intermediate or } \\ \text { Poor to intermediate }\end{array}$
Poor $\quad(n=494)$
$\quad(n=85)$

\section{B Women $(n=1,691)$}

\begin{tabular}{|c|c|}
\hline Ultra-rapid or Rapid & $(n=37)$ \\
\hline $\begin{array}{r}\text { Normal or } \\
\text { ntermediate to normal }\end{array}$ & $(n=865)$ \\
\hline $\begin{array}{l}\text { Intermediate or } \\
\text { Poor to intermediate }\end{array}$ & $(n=681)$ \\
\hline Poor & $(n=108)$ \\
\hline
\end{tabular}
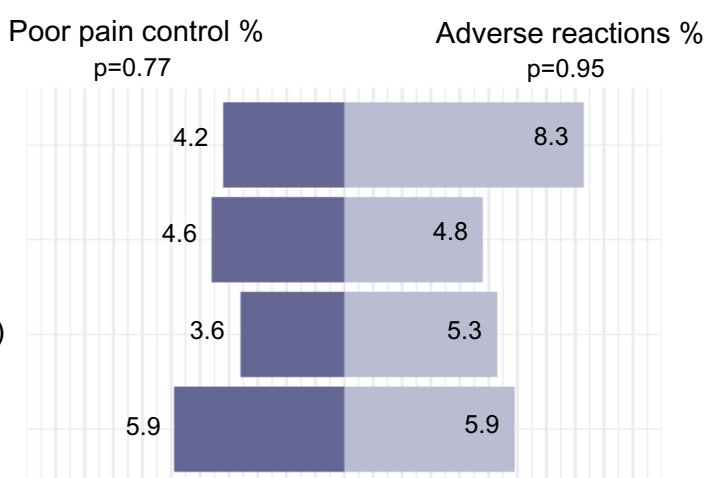

Poor pain control \% $\quad$ Adverse reactions \% $p=0.040 \quad p=0.041$

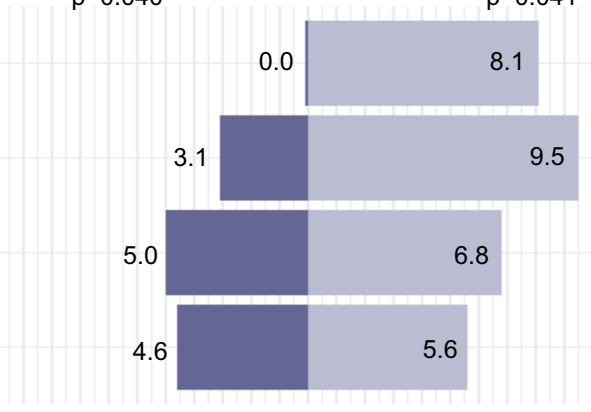

Figure 2 Adverse reactions and poor pain control following the prescription of codeine and/or tramadol by sex among men (A) and women (B) who were not prescribed CYP2D6 inhibitors $(n=2,877)$. 
prescribed only tramadol. Future research may investigate further sex differences in the associations between adverse reactions and poor pain control related to opioid use and CYP2D6 phenotypes.

\section{Discussion}

In the current study, participants with a CYP2D6 ultrarapid metabolizer phenotype experienced higher rates of adverse reactions, while participants with a CYP2D6 poor metabolizer phenotype experienced higher rates of poor pain control. These differences were driven primarily by women, suggesting that sex modified the association between CYP2D6 phenotype and these outcomes.

Our results are consistent with the results of previous research suggesting associations between CYP2D6 phenotypes and response to opioid use. ${ }^{10}$ However, the proportion of individuals experiencing adverse reactions and poor pain control among those with CYP2D6 poor and ultra-rapid metabolizer phenotypes differed from the proportions reported by previous research. For example, VanderVaart et $\mathrm{al}^{8}$ reported that $100 \%$ of CYP2D6 poor metabolizers experienced no analgesia, whereas $67 \%$ of ultra-rapid metabolizers reported adverse reactions. Here, we documented noticeably different results. Overall, 5.2\% (ie, 10 out of 193) of poor metabolizers experienced poor pain control, and $8.2 \%$ (ie, 5 out of 61 ) of ultra-rapid and rapid metabolizers reported adverse reactions. Our study sample consisted of persons recruited from the Mayo Clinic primary care practice, and the characteristics of the population broadly reflect the characteristics of persons residing in this community. ${ }^{20}$ Therefore, our study population may better reflect the experience of persons residing in the community. However, in the study conducted by VanderVaart et al, participants responded to a visual ana$\log$ scale while they were in the hospital, whereas we reviewed electronic health records for indications of poor pain control retrospectively. Differences in study populations and in the measurement of adverse reactions and poor pain control likely account for the differences in outcomes between these studies.

To address differences in CYP2D6 classification between studies, we also investigated associations between CYP2D6 activity scores and response to opioids, and found that these associations corroborated the results using CYP2D6 phenotype categorizations reported in the current study and in previous research. ${ }^{8,10}$ However, we note that higher CYP2D6 activity scores were not significantly associated with adverse reactions or poor pain control. Despite our large sample size, the relatively small number of outcomes may have resulted in an inability to detect a statistically significant association. Future studies should be powered specifically to investigate CYP2D6 activity scores among individuals who experienced poor pain control and/or adverse reactions related to opioid use.

While not statistically significant, our findings revealed a trend of incremental increase in the risk of adverse reactions and incremental decrease in the risk of poor pain control from individuals with CYP2D6 poor to ultra-rapid or rapid metabolizer status. This trend reached statistical significance among female (but not male) participants. We also found that, among normal and intermediate to normal metabolizers, a larger proportion of women experienced adverse reactions relative to men. This result was not detected in assessing an interaction or difference in the trends. Previous studies indicate that response to opioid medications differs in men and women, ${ }^{27}$ and men and women are known to metabolize opioids differently. ${ }^{28}$ In particular, higher CYP2D6 activity among extensive metabolizers has been observed in women compared to men, ${ }^{29}$ suggesting that underlying biologic differences may contribute to differences in CYP2D6 activity. Sex hormones have been studied as potential modifiers of CYP2D6 activity. However, menstrual phase and use of oral contraceptives have not been associated with CYP2D6 activity in several previous studies. ${ }^{30-32}$ CYP2D6 activity does increase in pregnant women, and pregnancy changes in liver tissue small heterodimer partner expression have been hypothesized to increase CYP2D6 activity in these women. ${ }^{33}$ Therefore, further studies are needed to understand whether underlying biologic differences between men and women might account for the associations we observed in this study.

Finally, our results indicated that older individuals (compared to younger individuals) and Blacks (compared to Whites) were more likely to experience adverse reactions related to opioids and poor pain control, respectively. These results are consistent with previous research suggesting that these characteristics may affect a person's response to opioids. In addition, previous findings revealed that Whites experienced more nausea and vomiting related to opioid use than Blacks. ${ }^{34}$ We did not replicate these results. Unfortunately, our numbers were too small to allow for sub-analyses within these populations, and our results are likely conservative estimates of the effects of covariates on opioid response. Future studies should consider the effect of these characteristics on the associations between CYP2D6 metabolism and adverse effects or poor pain control related to codeine and tramadol use. 
Our study has several strengths, including a large sample size and an ability to extract information directly from patient medical records shortly after the time of the codeine or tramadol prescription. However, our study also has several important limitations that are common to retrospective epidemiological research studies. First, our review of electronic health records for indication of adverse reactions and poor pain control relied on medical notes, such that we could not assess patterns of opioid use not documented in the medical notes (ie, patient self-medicating lower doses of prescribed opioid or not taking the opioid at all without informing their health-care provider), or adverse reactions not reported to health-care providers. Previous research has verified the appropriateness of chart review for the detection of adverse drug reactions. ${ }^{35}$ However, future research may employ more than one methodology to assess the effectiveness and adverse reactions related to opioid use, such as active follow-up of patients prescribed opioid medications. Second, tramadol is an atypical opioid as it is involved in partial $\mu$ agonist activity in addition to central gamma amino butyric acid (GABA), catecholamine and serotonergic activities. We did not assess (and therefore could not control for) the specific pathways causing pain relief in the patients prescribed tramadol (eg, whether patient obtained pain relief due to a serotonin-norepinephrine reuptake inhibitor effect). Future research may investigate interactions between sex and CYP2D6 phenotypes in opioids with different pathways. Third, some participants may have been prescribed opioids in conjunction with other medications, making it difficult to causally attribute the occurrence of adverse reactions to a particular opioid prescription. Fourth, despite our large sample size, the number of participants with an ultra-rapid metabolizer phenotype was limited, and our study may have been underpowered to detect clinically meaningful associations. Future research may attempt to replicate the observed trends. Finally, the limited number of persons with each CYP2D6 phenotype in our study required us to collapse phenotype categories for analysis. The reported results most likely represent a conservative estimate of associations between CYP2D6 phenotypes and response to opioids. Future research may investigate sex differences in response to opioids among persons with less common CYP2D6 phenotypes (eg, rapid metabolizers).

In summary, using a large sample of patients and different approaches to assess CYP2D6 metabolizing status (ie, phenotype categorization and activity scores), we replicated and extended the findings of previous research indicating associations between CYP2D6 phenotypes and activity scores with response to opioids. In addition, the associations between CYP2D6 phenotypes and response to opioid use were stronger in women than men, suggesting that future studies may benefit from considering sex differences in response to medications.

\section{Acknowledgment}

This work was supported by the Mayo Clinic Specialized Center of Research Excellent on Sex Differences, the Mayo Clinic Center for Individualized Medicine, the Robert D. and Patricia E. Kern Center for the Science of Health Care Delivery, and the National Institutes of Health grants [U19 GM61388 (The Pharmacogenomics Research Network), R01 GM28157, U01 HG005137, R01 GM125633, R01 AG034676 (The Rochester Epidemiology Project)], and [U01 HG06379 and U01 HG06379 Supplement (The Electronic Medical Record and Genomics (eMERGE) Network)].

\section{Disclosure}

Dr John Logan Black III has a patent Selection of Psychotropics with royalties paid to Myriad, also royalties, stock ownership from OneOme LLC. The authors report no other conflicts of interest in this work.

\section{References}

1. Centers for Disease Control and Prevention. 2018 Annual surveillance report of drug-related risks and outcomes - United States; 2018 [Updated August 31, 2018]. Available from: https://www.cdc.gov/dru goverdose/pdf/pubs/2018-cdc-drug-surveillance-report.pdf. Accessed March 7, 2020.

2. Abdel Shaheed C, Maher CG, Williams KA, Day R, McLachlan AJ. Efficacy, tolerability, and dose-dependent effects of opioid analgesics for low back pain: a systematic review and meta-analysis. JAMA Intern Med. 2016;176(7):958-968. doi:10.1001/jamainternmed.2016.1251

3. Kalso E, Edwards JE, Moore RA, McQuay HJ. Opioids in chronic non-cancer pain: systematic review of efficacy and safety. Pain. 2004;112(3):372-380. doi:10.1016/j.pain.2004.09.019

4. Becker JB, Chartoff E. Sex differences in neural mechanisms mediating reward and addiction. Neuropsychopharmacology. 2019;44 (1):166-183. doi:10.1038/s41386-018-0125-6

5. Gaedigk A, Ingelman-Sundberg M, Miller NA, Leeder JS, WhirlCarrillo M, Klein TE. The pharmacogene variation (PharmVar) consortium: incorporation of the human cytochrome P450 (CYP) allele nomenclature database. Clin Pharmacol Ther. 2018;103(3):399-401. doi:10.1002/cpt.910

6. Kirchheiner J, Schmidt H, Tzvetkov M, et al. Pharmacokinetics of codeine and its metabolite morphine in ultra-rapid metabolizers due to CYP2D6 duplication. Pharmacogenomics J. 2007;7(4):257-265. doi:10.1038/sj.tpj.6500406

7. Crews KR, Gaedigk A, Dunnenberger HM, et al. Clinical pharmacogenetics implementation consortium guidelines for cytochrome P450 2D6 genotype and codeine therapy: 2014 update. Clin Pharmacol Ther. 2014;95(4):376-382. doi:10.1038/clpt.2013.254

8. VanderVaart S, Berger H, Sistonen J, et al. CYP2D6 polymorphisms and codeine analgesia in postpartum pain management: a pilot study. Ther Drug Monit. 2011;33(4):425-432. doi:10.1097/FTD.0b013e3 $182272 \mathrm{~b} 10$ 
9. Smith DM, Weitzel KW, Elsey AR, et al. CYP2D6-guided opioid therapy improves pain control in CYP2D6 intermediate and poor metabolizers: a pragmatic clinical trial. Genet Med. 2019;21 (8):1842-1850. doi:10.1038/s41436-018-0431-8

10. St Sauver JL, Olson JE, Roger VL, et al. CYP2D6 phenotypes are associated with adverse outcomes related to opioid medications. Pharmgenomics Pers Med. 2017;10:217-227. doi:10.2147/PGPM. S136341

11. Caudle KE, Dunnenberger HM, Freimuth RR, et al. Standardizing terms for clinical pharmacogenetic test results: consensus terms from the clinical pharmacogenetics implementation consortium (CPIC). Genet Med. 2017;19(2):215-223. doi:10.1038/gim.2016.87

12. Ruano G, Kost JA. Fundamental considerations for genetically-guided pain management with opioids based on CYP2D6 and OPRM1 polymorphisms. Pain Physician. 2018;21(6): E611-E621. doi:10.36076/ppj.2018.6.E611

13. Ji Y, Skierka JM, Blommel JH, et al. Preemptive pharmacogenomic testing for precision medicine: a comprehensive analysis of five actionable pharmacogenomic genes using next-generation DNA sequencing and a customized CYP2D6 genotyping cascade. $J \mathrm{Mol}$ Diagn. 2016;18(3):438-445. doi:10.1016/j.jmoldx.2016.01.003

14. Fillingim RB, Gear RW. Sex differences in opioid analgesia: clinical and experimental findings. Eur $J$ Pain. 2004;8(5):413-425. doi:10.1016/j.ejpain.2004.01.007

15. Pisanu C, Franconi F, Gessa GL, et al. Sex differences in the response to opioids for pain relief: a systematic review and meta-analysis. Pharmacol Res. 2019;148:104447. doi:10.1016/j.phrs.2019.104447

16. Doyle HH, Murphy AZ. Sex-dependent influences of morphine and its metabolites on pain sensitivity in the rat. Physiol Behav. 2018;187:32-41. doi:10.1016/j.physbeh.2017.11.030

17. Averitt DL, Eidson LN, Doyle HH, Murphy AZ. Neuronal and glial factors contributing to sex differences in opioid modulation of pain Neuropsychopharmacology. 2019;44(1):155-165. doi:10.1038/ s41386-018-0127-4

18. Bielinski SJ, Olson JE, Pathak J, et al. Preemptive genotyping for personalized medicine: design of the right drug, right dose, right time-using genomic data to individualize treatment protocol. Mayo Clin Proc. 2014;89(1):25-33. doi:10.1016/j.mayocp.2013.10.021

19. Bielinski SJ, St Sauver JL, Olson JE, et al. Cohort profile: the right drug, right dose, right time: using genomic data to individualize treatment protocol (RIGHT protocol). Int J Epidemiol. 2019. doi:10.1093/ije/dyz123

20. Olson JE, Ryu E, Johnson KJ, et al. The Mayo Clinic Biobank: a building block for individualized medicine. Mayo Clin Proc. 2013;88(9):952-962. doi:10.1016/j.mayocp.2013.06.006

21. St Sauver JL, Grossardt BR, Yawn BP, et al. Data resource profile: the Rochester epidemiology project (REP) medical records-linkage system. Int $J$ Epidemiol. 2012;41(6):1614-1624. doi:10.1093/ije/ dys 195

22. Black JL 3rd, Walker DL, O'Kane DJ, Harmandayan M. Frequency of undetected CYP2D6 hybrid genes in clinical samples: impact on phenotype prediction. Drug Metab Dispos. 2012;40(1):111-119. doi:10.1124/dmd.111.040832
23. Kramer WE, Walker DL, O'Kane DJ, et al. CYP2D6: novel genomic structures and alleles. Pharmacogenet Genomics. 2009;19 (10):813-822. doi:10.1097/FPC.0b013e3283317b95

24. U.S. Food and Drug Administration. Drug development and drug interactions: table of substrates, inhibitors and inducers. 2017. https://www. fda.gov/drugs/drug-interactions-labeling/drug-development-and-druginteractions-table-substrates-inhibitors-and-inducers\#table3-2. Accessed March 7, 2020.

25. Drewes AM, Jensen RD, Nielsen LM, et al. Differences between opioids: pharmacological, experimental, clinical and economical perspectives. Br J Clin Pharmacol. 2012;75(1):60-78. doi:10.1111/ j.1365-2125.2012.04317.x

26. National Institues of Health, Office of Research on Women's Health. $\mathrm{NIH}$ policy on sex as a biological variable. Available from: https:// orwh.od.nih.gov/sex-gender/nih-policy-sex-biological-variable. Accessed March 7, 2020.

27. Fullerton EF, Doyle HH, Murphy AZ. Impact of sex on pain and opioid analgesia: a review. Curr Opin Behav Sci. 2018;23:183-190. doi:10.1016/j.cobeha.2018.08.001

28. Franconi F, Campesi I. Pharmacogenomics, pharmacokinetics and pharmacodynamics: interaction with biological differences between men and women. Br J Pharmacol. 2014;171(3):15. doi:10.1111/ bph.2014.171.issue-3

29. Tamminga WJ, Wemer J, Oosterhuis B, et al. CYP2D6 and CYP2C19 activity in a large population of Dutch healthy volunteers: indications for oral contraceptive-related gender differences. Eur J Clin Pharmacol. 1999;55(3):177-184. doi:10.1007/s002280050615

30. Labbe L, Sirois C, Pilote S, et al. Effect of gender, sex hormones, time variables and physiological urinary $\mathrm{pH}$ on apparent CYP2D6 activity as assessed by metabolic ratios of marker substrates. Pharmacogenetics. 2000;10(5):425-438. doi:10.1097/00008571200007000-00006

31. Kashuba ADM, Nafziger AN, Kearns GL, et al. Quantification of intraindividual variability and the influence of menstrual cycle phase on CYP2D6 activity as measured by dextromethorphan phenotyping. Pharmacogenetics. 1998;8(5):403-410. doi:10.1097/00008571199810000-00005

32. McCune JS, Lindley C, Decker JL, et al. Lack of gender differences and large intrasubject variability in cytochrome P450 activity measured by phenotyping with dextromethorphan. J Clin Pharmacol. 2001;41(7):723-731. doi:10.1177/00912700122010627

33. Pan X, Ning M, Jeong H. Transcriptional regulation of CYP2D6 expression. Drug Metab Dispos. 2017;45(1):42-48. doi:10.1124/ dmd.116.072249

34. Cepeda MS, Farrar JT, Baumgarten M, Boston R, Carr DB, Strom BL. Side effects of opioids during short-term administration: effect of age, gender, and race. Clin Pharmacol Ther. 2003;74 (2):102-112. doi:10.1016/S0009-9236(03)00152-8

35. Miguel A, Azevedo LF, Lopes F, Freitas A, Pereira AC. Methodologies for the detection of adverse drug reactions: comparison of hospital databases, chart review and spontaneous reporting. Pharmacoepidemiol Drug Saf. 2013;22(1):98-102. doi:10.1002/pds. v22.1

Pharmacogenomics and Personalized Medicine

Dovepress

\section{Publish your work in this journal}

Pharmacogenomics and Personalized Medicine is an international, peer-reviewed, open access journal characterizing the influence of genotype on pharmacology leading to the development of personalized treatment programs and individualized drug selection for improved safety, efficacy and sustainability. This journal is indexed

on the American Chemical Society's Chemical Abstracts Service (CAS). The manuscript management system is completely online and includes a very quick and fair peer-review system, which is all easy to use. Visit http://www.dovepress.com/testimonials.php to read real quotes from published authors. 